\title{
Ciencia Abierta, un modelo por definir con muchos retos por delante
}

\author{
Ernest Abadal \\ Universitat de Barcelona \\ abadal@ub.edu \\ https://orcid.org/0000-0002-9151-6437
}

\section{Open science, an undefined model with many challenges}

\begin{abstract}
RESUMEN
La ciencia abierta es una manera de concebir la investigación científica que se basa en el trabajo colaborativo, en la apertura y transparencia de todas las fases de la investigación (recogida de datos, revisión por expertos, difusión, evaluación, etc.) y también en la aproximación de la ciencia a la sociedad. De todas formas, es un ámbito que aún se está perfilando y que es poco conocido por parte de la mayoría de los investigadores. El objetivo del texto es presentar los diversos estudios que se publican en el número monográfico y que se centran en tres grandes ámbitos: la ciencia abierta, los datos de investigación y el acceso abierto. En segundo lugar, también se describen los cuatro principales retos e incertidumbres que, en estos momentos, amenazan el crecimiento e implantación de la ciencia abierta: liderado por las élites, velocidades distintas en sus componentes, riesgos de monopolio y pocas políticas de promoción.
\end{abstract}

PALABRAS CLAVE

Ciencia abierta; Acceso abierto; Datos de investigación; Comisión Europea; Políticas

\section{ABSTRACT}

Open science is an approach to scientific research based on collaboration, on openness and transparency at each stage of the research process (including, data collection, peer review, dissemination, evaluation, etc.) and on the enhancement of its accessibility to society. Nevertheless, it is an area that is still being profiled and that isn't well-known by researchers. The objective of our text is to present the studies published in this monographic issue and we can match them up in three topics: open science, research data and open access. After that, we want to point on four main challenges and uncertainties that, at this moment, can be a threat to the growth and implantation of open science: there are a top-down leadership, different speeds in their components, serious risk of a monopoly and a lack of promotion policies.

\section{KEYWORDS}

Open science; Open access; Research data; European Comission; Policies 


\section{Qué es la ciencia abierta}

La ciencia abierta es una manera de concebir la investigación científica que se basa en el trabajo colaborativo, en la apertura y transparencia de todas las fases de la investigación (recogida de datos, revisión por expertos, difusión, evaluación, etc.) y también en la aproximación de la ciencia a la sociedad. La ciencia abierta supone una transformación radical de la manera de llevar a cabo la investigación científica y de cómo transformar su sistema de evaluación, en síntesis, todo un cambio de paradigma respecto del actual sistema (Anglada y Abadal, 2018) (Packer y Santos, 2019).

Hasta el momento, se trata de un concepto amplio bajo el cual caben muchos elementos, empezando por el acceso abierto a las publicaciones -impulsado desde inicios de 2000-, los datos científicos abiertos - solicitados en proyectos europeos desde 2014-, y continuando por elementos menos conocidos como las revisiones abiertas (open peer review), la ciencia ciudadana (citizen science) o los nuevos modelos de evaluación. Cada uno de estos elementos ha tenido una evolución propia, a distinta velocidad y con poca interrelación. Así pues, la ciencia abierta se entiende de momento más bien como un concepto "paraguas" (umbrella term, en inglés), en el cual la potencia se encuentra más bien en las partes que en el todo.

Quien ha impulsado este nuevo modelo y lo ha situado en la agenda temática internacional ha sido, sin duda, la Comisión Europea con el documento Digital Science in Horizon 2020 (2013) y otros más que le han seguido, en especial las recomendaciones sobre acceso y preservación de la información científica (Comisión Europea, 2018). Esta misma institución ha creado un portal sobre ciencia abierta (Comisión Europea, 2019) que recoge los documentos y las novedades sobre esta cuestión.

La bibliografía sobre ciencia abierta ya es cada vez más amplia y, por tanto, difícil de resumir. Por ello, queremos destacar un artículo de revisión bibliográfica que repasa el concepto de ciencia abierta (Vicente y Martínez, 2018), la monografía de Bartling y Friesike (2014), una de las primeras sobre la cuestión, así como los materiales y documentos divulgativos que ha elaborado el proyecto FOSTER (2019). A partir de estas tres referencias se puede obtener una buena visión global sobre el concepto de ciencia abierta.

\section{Diversas caras y líneas de estudio}

En este número monográfico de Hipertext.net se propuso a académicos y profesionales el envío de trabajos de investigación originales relacionados con los elementos antes señalados (acceso abierto, datos abiertos, revisión abierta, nuevos modelos de evaluación, etc.) y también con las políticas para conseguir la implantación del nuevo modelo. El resultado final lo podemos agrupar en tres grandes ámbitos:

\section{a) Ciencia abierta}

De Filippo y D'Onofrio (2019) llevan a cabo un análisis de las políticas públicas existentes actualmente en siete países latinoamericanos para dar apoyo a la ciencia abierta y, de manera paralela, un estudio bibliométrico de las publicaciones científicas sobre esta temática.

Centrándose en las humanidades digitales, Rio Riande y Tóth-Czifra (2019) analizan como se pueden introducir procesos de apertura en este ámbito. En su caso se centran en el proyecto OpenMethods y analizan las posibilidades que ofrece para el desarrollo de unas humanidades digitales más abiertas.

\section{b) Datos de investigación}

Marín-Arraiza et al. (2019) se centran en el apoyo que se da a la gestión de datos desde las bibliotecas y realizan un análisis y valoración de las líneas de actuación en bibliotecas de Europa y América Latina.

Por su parte, Morales y Codina (2019) analizan los atributos de calidad fundamentales para la evaluación de la interfaz web de los repositorios de datos, una de las infraestructuras fundamentales para asegurar el éxito del cambio de modelo. El protocolo de análisis que proponen incluye indicadores sobre la interfaz de usuario, la localización, acceso y reutilización, conservación, difusión, evaluación y el compromiso institucional

\section{c) Acceso abierto}

La gran novedad en la difusión en abierto de la producción científica ha sido el Plan $\mathrm{S}$ puesto en marcha por agencias de financiación europeas para acelerar la transición al acceso abierto. Abadal et al. (2019) analizan los contenidos principales de esta propuesta, así como las reacciones y críticas que ha generado.

Para estas aproximaciones, se pueden leer las aportaciones de autores españoles y latinoamericanos que han tomado en consideración la ciencia abierta con amplitud de miras ya que tan tenido en cuenta no tan sólo la situación en Europa, gran impulsora del movimiento, sino también en América Latina. A pesar de tratarse de un fenómeno global es importante estudiar y saber interpretar los matices y orientaciones particulares que se dan en estas realidades geográficas tan distintas. 


\section{El futuro de la ciencia abierta}

Así pues, nos encontramos delante de un ámbito que aún se está perfilando y que es poco conocido (al menos, con precisión) por parte de la mayoría de los investigadores. A estas incertidumbres, típicas en los estadios iniciales de cualquier cambio de modelo, se le suman algunos retos o sombras que en estos momentos amenazan a su crecimiento e implantación. Vamos a destacar cuatro de ellos.

\section{a) Movimiento liderado por las élites}

Aunque existen propuestas pioneras en ciencia abierta que han sido impulsadas por investigadores particulares, en general, no se trata de una iniciativa generada a partir de la base, sino que el empuje ha sido propiciado por parte de los gestores de la investigación, en especial de la Dirección General de Investigación de la Comisión Europea. Hasta que no se consiga la asimilación y adopción de estos nuevos principios por parte de los investigadores será difícil avanzar mucho más. Esta es una de las tareas que debe ocupar la atención de los gestores de la investigación y de las agencias de financiación.

\section{b) Velocidades distintas}

Siguiendo el símil del concepto paraguas, hay que tener presente que cada uno de los elementos básicos que componen la ciencia abierta (ya sea el acceso abierto, los datos, la ciencia ciudadana, etc.) avanza a su propia velocidad (mucho más rápido el acceso abierto que los datos, por ejemplo). En algún caso, además, está aún por definir la estructura básica, como pasa con la evaluación de la ciencia, ámbito en el cual están por consensuar los indicadores que deberían aplicarse a la ciencia abierta.

\section{c) Riesgos de monopolio}

Mirowski es un autor que no cree que la ciencia abierta suponga ninguna revolución porque la ve como una nueva expresión del neoliberalismo. En su artículo The future(s) of open science (Mirowski, 2018) alerta del posible monopolio que puede instalarse en el nuevo escenario de la ciencia abierta. En su opinión, las empresas de servicios para la investigación se están comportando de manera parecida a como Facebook o Google han actuado en el ámbito de los servicios generales de internet. Es decir, estas empresas están integrando la diversidad de servicios que existían hasta ahora (bases de datos científicas, índices de impacto, gestión de datos de investigación, perfiles de autor, gestión de revistas, etc.) en grandes plataformas de apoyo a la investigación para obtener el dominio del mercado y reducir la competencia. Ciertamente, este es otro de los riesgos que se cierne sobre el desarrollo de la ciencia abierta y habrá que aprovechar la experiencia vivida en el sector de los servicios de internet para proponer medidas que impidan o dificulten la creación de entornos de casi-monopolio.

\section{d) Pocas políticas de apoyo}

En otro artículo (Abadal y Anglada, 2019) analizamos la situación de las políticas en Europa y en el texto de De Filippo (2019), antes comentado, se hace lo mismo con Latinoamérica. En ambos casos, no obstante, queda claro que aún no se han generalizado actuaciones ni programas políticos de alcance global (de ciencia abierta y no tan sólo sobre acceso abierto o datos abiertos) que impulsen el desarrollo y adopción de estas nuevas prácticas por parte de los investigadores.

En resumen, pues, la ciencia abierta supone una profunda transformación en el funcionamiento de la investigación científica, pero se trata de un modelo que aún no está totalmente definido ni perfilado, basado más bien en la acumulación de elementos y no en su plena conjunción e integración en un todo (el concepto paraguas, antes apuntado). Por otro lado, los retos y las incertidumbres que quedan por delante aún son notables (poca difusión entre investigadores, riesgos de monopolio, desarrollo de políticas de apoyo, etc.) y, por tanto, se va a necesitar un tiempo de asimilación y de práctica por parte de los investigadores para disiparlas.

A pesar de todo ello, esperemos que a medio plazo se consigan integrar todos estos elementos en una visión global y que su adopción generalizada suponga una auténtica revolución en la manera de investigar y de generar conocimiento científico.

\section{Referencias}

Abadal, E., y Anglada, L. (2019). Políticas de ciencia abierta en Europa. Zenodo. http://doi.org/10.5281/zenodo.3526654

Abadal, E., López-Borrull, À., Ollé-Castellà, C., y García-Grimau, F. (2019). El plan $S$ para acelerar el acceso abierto: contexto, retos y debate generado. Hipertext.net (19). https://doi.org/10.31009/hipertext.net.2019.i19.06

Anglada, L., y Abadal, E. (2018). ¿Qué es la ciencia abierta? Anuario ThinkEPI (12), 292-298. https://doi.org/10.3145/thinkepi.2018.43

Bartling, S., y Friesike, S. (2014). Towards Another Scientific Revolution. En: Bartling S., y Friesike S. (eds). Opening Science. Springer, Cham. https://doi.org/10.1007/978-3-319-00026-8

Comisión Europea (31 mayo 2018). Commission Recommendation (EU) $2018 / 790$ of 25 April 2018 on access to and preservation of scientific information. Official Journal of the European Union. Recuperado de https://eur-lex.europa.eu/legal-content/EN/TXT/PDF/?uri=CELEX:32018H0790\&from=EN

Comisión Europea (2019). Open Science. Recuperado de http://ec.europa.eu/research/openscience/index.cfm

De Filippo, D., y D'Onofrio, M. G. (2019). Alcances y limitaciones de la ciencia abierta en Latinoamérica: análisis de las políticas públicas y publicaciones científicas de la región. Hipertext.net (19). https://doi. org/10.31009/hipertext.net.2019.i19.03

Digital (2013). Digital science in Horizon 2020. Recuperado de https:// 
ec.europa.eu/digital-single-market/en/news/digital-science-horizon-2020

FOSTER (2019). The future of science is open. Recuperado de https:// www.fosteropenscience.eu

Marín-Arraiza, P., Puerta-Díaz, M., y Gregorio-Vidotti, S. (2019). Gestión de datos de investigación y bibliotecas: preservando los nuevos bienes científicos. Hipertext.net (19). https://doi. org/10.31009/hipertext.net.2019.i19.02

Mirowski, P. (2018). The future(s) of open science. Social Studies of Science, 48(2), 171-203. https://doi.org/10.1177/0306312718772086

Morales-Vargas, A., y Codina, L. (2019). Atributos de calidad web para repositorios de datos de investigación en universidades. Hipertext. net (19). https://doi.org/10.31009/hipertext.net.2019.i19.04

Packer, A., y Santos, S. (1 agosto 2019). Ciencia abierta y el nuevo modus operandi de comunicar la investigación - Parte I. SciELO en Perspectiva. Blog SciELO.org. Recuperado de https://blog.scielo.org/ es/2019/08/01/ciencia-abierta-y-el-nuevo-modus-operandi-de-comunicar-la-investigacion-parte-i

Río Riande, G. del, y Tóth-Czifra, E. (2019). OpenMethods o cómo abrir la investigación en Humanidades Digitales. Hipertext.net (19). https:// doi.org/10.31009/hipertext.net.2019.i19.05

Vicente-Saez, R., y Martínez-Fuentes, C. (2018). Open Science now: A systematic literature review for an integrated definition. Journal of Business Research. https://doi.org/10.1016/j.jbusres.2017.12.043

\section{CV}

Ernest Abadal. Catedrático de la Facultat de Información y Medios Audiovisuales de la Universitat de Barcelona. Director del Centro de Investigación en Información, Comunicación y Cultura. Dirige un proyecto de investigación sobre ciencia abierta en España. Es miembro del consejo asesor de diversas revistas científicas. https://fima.ub.edu/pub/abadal/

\section{ocm Observatorio de Cibermedios}

\section{https://observatoriocibermedios.upf.edu/}

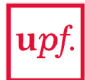

Universitat Pompeu Fabra

Departamento de Comunicación Grupo DigiDoc
El Observatorio de Cibermedios es una producción del Grupo de Investigación en Documentación Digital y Comunicación Interactiva (DigiDoc) del Departamento de Comunicación de la Universitat Pompeu Fabra.

El Observatorio de Cibermedios (OCM) forma parte del proyecto del Plan Nacional "Creación y contenido interactivo en la comunicación de información audiovisual: audiencias, diseño, sistemas y formatos". CS02015-64955-C4-2-R (MINECO/ FEDER), Ministerio de Economía y Competitividad (España). 